\title{
3D Models of Blood Flow in the Cerebral Vasculature S.Moore $^{a}$, T.David ${ }^{a}$, J.G.Chase $^{a}$, J.Arnold $^{a}$, J.Fink $^{b}$ \\ ${ }^{a}$ Center for Bioengineering, Department of Mechanical Engineering, University of Canterbury, Christchurch, New Zealand \\ ${ }^{b}$ Christchurch School of Medicine and Health Sciences, University of Otago, Christchurch, New Zealand
}

\begin{abstract}
The circle of Willis (CoW) is a ring-like arterial structure located in the base of the brain and is responsible for the distribution of oxygenated blood throughout the cerebral mass. To investigate the effects of the complex 3D geometry and anatomical variability of the CoW on the cerebral hemodynamics, a technique for generating physiologically accurate models of the CoW has been created using a combination of magnetic resonance data and computer aided design software. A mathematical model of the body's cerebral autoregulation mechanism has been developed and numerous computational fluid dynamics simulations performed to model the hemodynamics in response to changes in afferent blood pressure. Three pathological conditions were explored, namely a complete CoW, a fetal P1 and a missing A1. The methodology of the cerebral hemodynamic modelling is proposed with the potential for future clinical application in mind, as a diagnostic tool.
\end{abstract}

\section{Introduction}

The Circle of Willis (CoW) (Figures 1, 2,3) is a ring-like arterial structure located in the base of the brain and is responsible for the distribution of oxygenated blood throughout the cerebral mass. The Internal Carotid and Vertebral arteries (ICA's, VA's) supply blood into the circle and are termed afferent arteries (where a prefix 'L' or 'R' in front of an artery abbreviation indicates that segment on the left or right hand side of the CoW respectively). The Anterior, Middle and Posterior Cerebral arteries (ACA, MCA, PCA) transport blood away from the circle and are termed efferent arteries. The anastomosing arteries, namely the Anterior and Posterior Communicating arteries (ACoA, PCoA's) connect the efferent arteries, hence allowing blood to be re-routed to maintain oxygen supply to the the whole cerebral mass, should any afferent blood supply be reduced.

Among the general population, approximately 50\% have a complete CoW (Alpers et al. 1959), where possible variations can include underdeveloped or completely absent blood vessels. While an individual with one of these variations may suffer no ill effects under normal circumstances, the reduced ability for collateral blood flow via the circle of Willis compounded with certain pathological conditions can present a health risk, in particular the possibility of suffering an ischaemic stroke.

The importance of understanding the sensitivity of cerebral perfusion on circle of Willis geometry arises from the fact that in the western world stroke is the third largest cause of death after heart disease and cancer, and is the largest cause of serious long-term disability (Petty et al. 2000). The monetary costs associated with stroke care are considerable and it is projected that if the incidence of stroke continues to increase at the same rate it will more than triple in the next 30 years. There is thus a growing importance in the understanding of factors that increase the risk of stroke and how blood is distributed throughout the brain.

There has been a significant body of research performed on blood flow in the circle of Willis (Moorhead et al. 2004; Hillen et al. 1986; Cassot et al. 2000), treating the cerebral vasculature as a 1D structure and assuming Poiseuille flow. This approach however cannot capture the effects of the complex arterial geometry, in particular the effects of blood vessel junctions. Subsequent 2D models of the circle of Willis (Ferrandez et al. 2002; David et al. 2003) have improved the definition of the geometry, however to obtain more realistic hemodynamic data 3D models must be considered. Studies have been performed on 3D models of the circle of Willis (Cebral et al. 2003) generated from Magnetic Resonance Imaging (MRI) data, however, the 3D models neglected the effects of autoregulation on cerebral blood flow.

The body's cerebral autoregulation mechanism is the ability of the arterioles to vasoconstrict/dilate in response to changes in cerebral perfusion pressure over a certain range, thus maintaining a relatively constant cerebral blood flow. These effects are of particular importance when investigating how blood is redistributed via the circle of Willis. It should be noted that there have been numerous cerebral autoregulation models proposed (Ursino and Giulioni 2003; Ursino 1991; Olufsen et al. 2002), including models incorporated with a 
circle of Willis (Moorhead et al. 2004; Ferrandez et al. 2002; David et al. 2003). However, the combination of autoregulation with the more realistic 3D circle of Willis geometries remains a relatively unexplored field.

For the present study, a technique for creating the complex 3D geometries from magnetic resonance data and incorporating an autoregulation mechanism is presented. Numerical fluid simulations have been performed on three common pathological circle of Willis geometries, providing some basic results investigating the effect of geometry in response to alterations in afferent blood pressure. The methodology for the cerebral hemodynamic modelling is proposed with the potential for future clinical application as a diagnostic tool. The potential areas of use include the prediction of an individual's cerebral response to alterations in their afferent blood pressure, particularly during surgical procedures such as a carotid endarterectomy.

\section{Theory and Methodology}

\subsection{Anatomical Modelling}

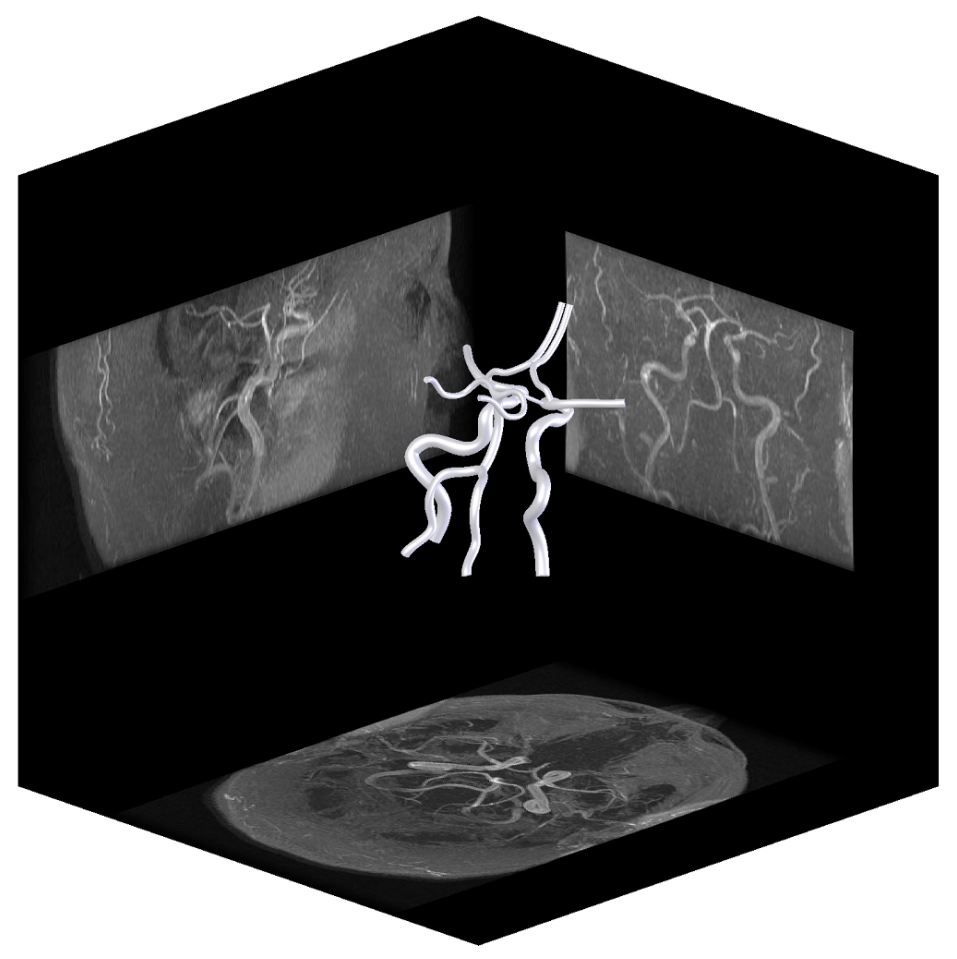

Figure 1: 3D Circle of Willis Geometry

The circle of Willis geometry has been generated using a combination of Computer Aided Design (CAD) software and Magnetic Resonance Angiography (MRA) data. Initially, three scaled maximum intensity projections (MIP's) on mutually orthogonal planes were taken from a single time of flight (TOF) MRA scan and imported into the CAD package Solidworks (Figure 1). Splines were subsequently traced over the arterial branches of interest on any two of the MIP's and projected normal to the plane on which they were created, providing curves in 3D space and a resulting generic skeletal circle of Willis structure. Circular cross sections were then lofted along the 3D curves and the junctions between arterial segments also lofted together to complete the 3D model. Generating the geometry using this technique, as opposed to direct segmentation and reconstruction from MRI data produces a reasonably accurate physiological approximation to the cerebral vasculature, with only the loss of the finer topological details of the surface of the arterial walls. Compared to the lengths and diameters of the arterial branches however, these topological features will have a minimal effect in the main efferent blood supply.

As can be observed from Figure 1 the efferent arteries have been truncated a short distance downstream of the circle, before their first bifurcation. The reasoning for terminating the model is twofold. Firstly, the 
interest for the present study is how blood is redistributed throughout the circle of Willis in response to alterations in afferent blood pressure. Therefore, continuing the efferent arteries further would not provide any further information on this point. Secondly, limiting the model size will save on computational size and solution time. It is important however that the downstream boundaries be located far enough away that from arterial junctions such that the efferent velocity profiles be fully developed.

To represent the effects of the arteriole, venous and capillary beds, eliminated from the model by the choice of efferent artery termination, the approach of using a porous block, proposed by (Ferrandez et al. 2002; David et al. 2003), at the termination of each efferent artery has been implemented. The permeability of the porous blocks can be set to provide a resistance to the flow in much the same way as the vascular beds, hence realistic representations of the hemodynamics can still be achieved.

Considering both the geometrical variability and number of the arterial segments comprising the circle of Willis, investigating the sensitivity of the hemodynamics to different geometries could result in a large number of possible simulations. Three of the more common pathological conditions of the circle of Willis (Alpers et al. 1959) were chosen for study, namely a complete circle of Willis, a fetal P1 and a missing A1. The difference between a Fetal P1 configuration (Figure 2) compared to a complete circle is that one of the $\mathrm{P} 1$ segments of a PCA is greatly reduced in size, while the PCoA ipsilateral to that P1 is increased in size. In the case of a Missing A1 (Figure 3), one of the A1 segments from an ACA is either completely absent from the cerebral vasculature, or is decreased to such a size that there would be negligible blood flow through it. Furthermore, in this condition there is essentially no ACoA, rather the remaining A1 bifurcates into the two A2 segments.

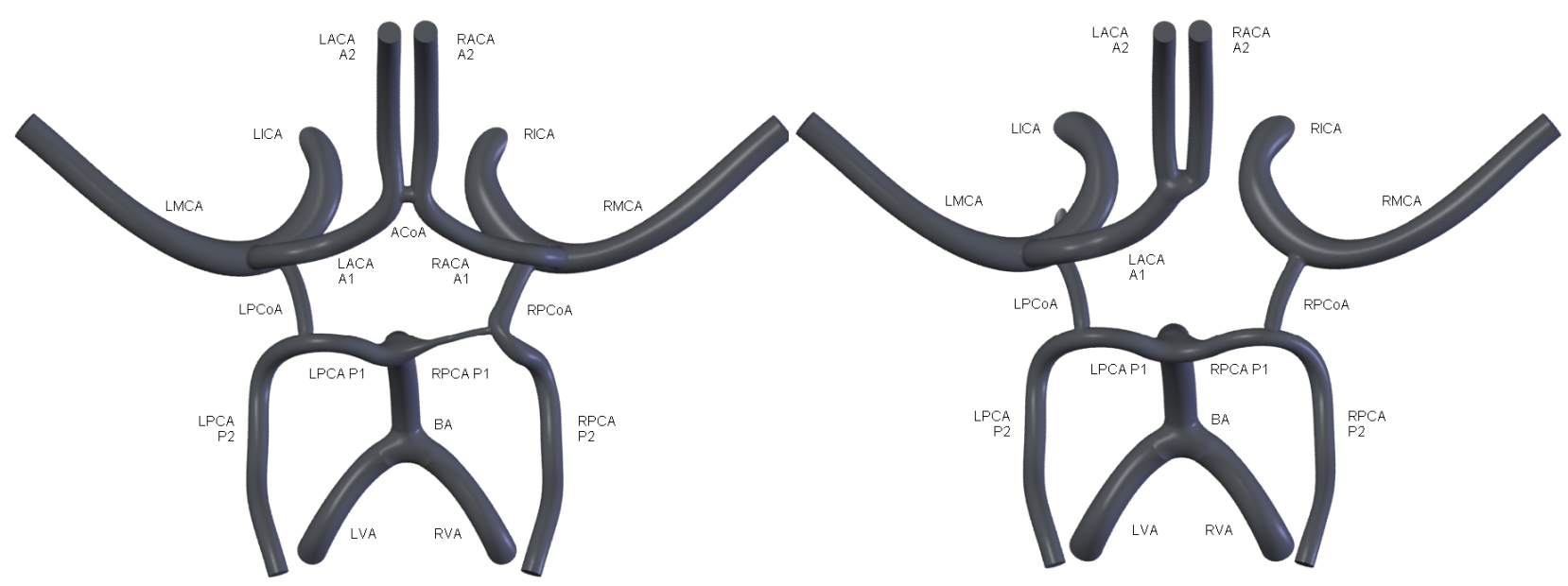

Figure 2: Fetal P1

Figure 3: Missing A1

As the flow through the blood vessels depends much more strongly on the diameter as opposed to the length, it was decided to use only a single generic 3D skeletal circle of Willis structure for all of the geometries and alter only the diameters.

The diameters of the various arterial segments of the circle of Willis were obtained from a retrospective population study of MRA scans. The diameters were measured using an in-house software package (Volume Estimation Toolkit [VET], Canterbury District Health Board), which allows the various slices from a given MRA TOF scan to be read and by interactively dragging a cursor between points of interest, obtain measurements of the vessels. For the complete circle of Willis, 13 cases were examined, for the fetal P1 pathological condition, 14 cases were examined and for the missing A1 pathological condition 10 cases were examined. The mean values for the diameters used to create each model and their standard deviations are shown in Tables 1 where the terms ipsilateral and contralateral refer to the same side and opposite sides of the $\mathrm{CoW}$ as the pathological condition respectively. Each 3D mesh generated comprised of approximately 1 million tetrahedral volumes with a nodal spacing ranging in size from $0.2 \mathrm{~mm}$ in the smaller efferent arteries and communicating arteries to $0.35 \mathrm{~mm}$ in the larger afferent arteries. 
Table 1: Circle of Willis Measurements

\begin{tabular}{llcccccc}
\hline & & \multicolumn{2}{c}{ Complete CoW } & \multicolumn{2}{c}{ Fetal P1 } & \multicolumn{2}{c}{ MissingA1 } \\
\hline Artery & & Diameter & Std Dev & Diameter & Std Dev & Diameter & Std Dev \\
& & $m m$ & $m m$ & $m m$ & $m m$ & $m m$ & $m m$ \\
\hline ACA - A1 & ipsilateral & 2.33 & 0.22 & 2.16 & 0.40 & - & - \\
& contralateral & 2.33 & 0.22 & 2.38 & 0.41 & 2.38 & 0.41 \\
ACA - A2 & & 2.40 & 0.31 & 2.50 & 0.31 & 2.55 & 0.15 \\
MCA - M1 & ipsilateral & 2.86 & 0.17 & 2.95 & 0.29 & 2.91 & 0.48 \\
& contralateral & 2.86 & 0.17 & 2.99 & 0.20 & 2.88 & 0.28 \\
PCA - P1 & ipsilateral & 2.13 & 0.25 & 1.55 & 0.40 & 1.88 & 0.41 \\
& contralateral & 2.13 & 0.25 & 2.99 & 0.46 & 2.19 & 0.39 \\
PCA - P2 & ipsilateral & 2.10 & 0.21 & 2.11 & 0.20 & 2.05 & 0.37 \\
& contralateral & 2.10 & 0.21 & 2.08 & 0.24 & 2.16 & 0.30 \\
ACoA & & 1.47 & 0.17 & 1.63 & 0.43 & - & - \\
PCoA & ipsilateral & 1.45 & 0.31 & 2.01 & 0.39 & 1.74 & 0.39 \\
BA - B1 & contralateral & 1.45 & 0.31 & 2.08 & 0.24 & 1.66 & 0.58 \\
BA - B2 & & 3.17 & 0.51 & 2.77 & 0.40 & 2.93 & 0.36 \\
ICA & & 3.29 & 0.44 & 2.86 & 0.56 & 3.31 & 0.34 \\
\hline
\end{tabular}

\subsection{Hemodynamic Modelling}

Blood flow through the circle of Willis is modelled as unsteady, incompressible and viscous. Therefore, the governing equations to be solved are the continuity equation:

$$
\int_{A} \rho \mathbf{u} \cdot d \mathbf{A}=0
$$

and the momentum equation:

$$
\int_{V} \frac{\partial \mathbf{u}}{\partial t}+\int_{A} \rho \mathbf{u u} \cdot d \mathbf{A}=-\int_{A} p \mathbf{I} d \mathbf{A}+\int_{A} \eta(\bar{\epsilon}) \bar{\epsilon} \cdot d \mathbf{A}+\int_{V} \mathbf{F} d V
$$

where $\mathbf{u}$ is the velocity vector, $\rho$ is the blood density, $p$ is the pressure, $\eta$ is the fluid viscosity, $\mathbf{F}$ represents any additional momentum sink terms to be incorporated and $\bar{\epsilon}$ is the strain rate tensor, defined as:

$$
\bar{\epsilon}=\frac{1}{2}\left(\nabla \mathbf{u}+\nabla \mathbf{u}^{T}\right)
$$

It is well known that blood is a non-Newtonian fluid due to its shear thinning and viscoelasticity. Furthermore, recent studies (Chen and Lu 2004) suggest that despite the relatively high shear rates through larger arteries, the hemodynamics are still affected by the non-Newtonian nature of the blood, especially where the vessels follow tortuous paths, such those in the circle of Willis. To account for the non-Newtonian nature of blood the Carreau-Yasuda model has been implemented using the constitutive parameters taken from (Gijsen et al. 1999). This model accounts for the red blood cell aggregation and deformation at low and high shear rates respectively and is defined:

$$
\frac{\eta-\eta_{\infty}}{\eta_{0}-\eta_{\infty}}=\left(1+(\lambda \dot{\gamma})^{a}\right)^{\frac{n-1}{a}}
$$

where $\eta_{0}$ is the zero-shear viscosity and $\eta_{\infty}$ the infinite shear viscosity, taken as 0.022 Pa.s and 0.0022 Pa.s respectively. The remaining constitutive parameters $\lambda, a, n$ are taken as $0.11 s, 0.644$ and 0.392 respectively. $\dot{\gamma}$ is the strain rate magnitude, derived from the second invariant of the strain rate tensor, which for an incompressible fluid becomes:

$$
\dot{\gamma}=2 \sqrt{\bar{\epsilon}_{i j} \bar{\epsilon}_{i j}}
$$

The last term in (2), representing any additional momentum sink terms, is used to represent the effects of the porous blocks, placed at the terminations of the efferent arteries. The momentum sink contributes to 
the pressure gradient in the porous block creating a pressure drop that is proportional to the blood velocity. For the case of a simple homogeneous porous media, the momentum sink is defined as:

$$
\mathbf{F}=-\left(\frac{\eta}{k} \mathbf{u}+C \frac{1}{2} \rho|\mathbf{u}| \mathbf{u}\right)
$$

where $k$ is the permeability and $C$ is the inertial resistance factor. In laminar flows, as is the case assumed for the present study, $C$ can be considered to be zero, leaving only the first term in (6).

\subsection{Cerebral Autoregulation Modelling}

In order to simulate realistic collateral flow through the circle of Willis in response to alterations in afferent blood pressure, the implementation of a physiologically realistic cerebral autoregulation mechanism is of crucial importance. The two important features of the autoregulation mechanism that need to be replicated are the autoregulation curve (Figure 4(a)) and the autoregulation dynamics (Figure 4(b)).

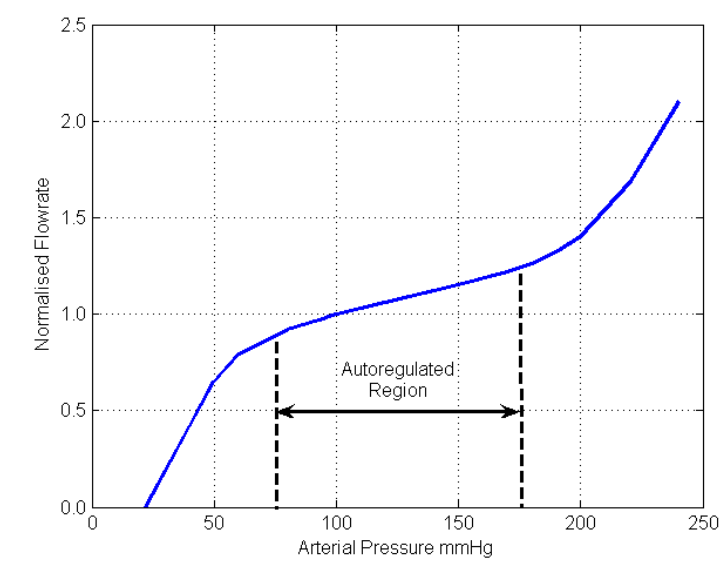

(a)

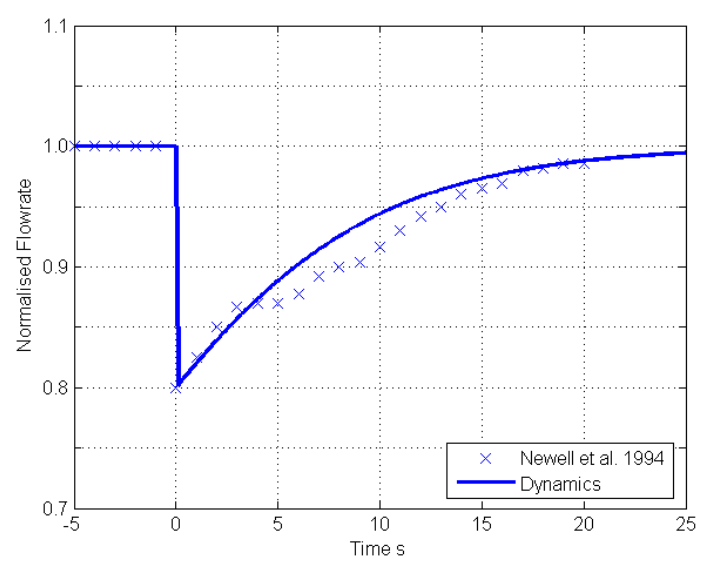

(b)

Figure 4: (a) Autoregulation Curve and (b) Autoregulation Dynamics

The autoregulation curve (Guyton and Hall 2000) assumes that in the autoregulated range of approximately $75-175 \mathrm{mmHg}$ the overall steady state blood flowrate through the brain is a weakly linear function of mean arterial blood pressure. Outside this range of arterial pressure, blood flow is completely unregulated. To replicate the linear region of the autoregulation a model relating blood flowrate to arterial pressure (Keener and Sneyd 1998) is defined:

$$
Q_{R e f}=\frac{1}{1+A\left[O_{2}\right]_{A}}\left(M A+\frac{P_{A}-P_{V}}{R_{0}}\right)
$$

where $Q_{R e f}$ is the steady state blood flowrate, $\left[\mathrm{O}_{2}\right]_{A}$ is the arterial oxygen concentration, $M$ is the metabolic rate, $P_{A}$ and $P_{V}$ arterial and venous pressures respectively and $A$ and $R_{0}$ are scaling parameters chosen so that (7) fits the curve in Figure 4(a). The autoregulation dynamics have been modelled as first order and based on the results of thigh cuff tests performed by (Newell et al. 1994). The arterioles responsible for vaso-dilation/constriction alter the resistance of the cerebrovascular bed to blood flow. This effect can be reproduced in the simulations by altering the permeability of the porous blocks, where each porous block can independently alter its permeability to achieve its required blood flow through the corresponding efferent artery. Furthermore, upper and lower limits can be placed on the permeability corresponding to the upper and lower arterial pressures of the autoregulated range. The autoregulation dynamics are modelled by a first order ODE for the permeability $(k)$ defined:

$$
\tau \frac{d k}{d t}=K_{p} \frac{Q_{R e f}-Q}{Q_{R e f}}+K_{i} \int_{0}^{t} \frac{Q_{R e f}-Q}{Q_{R e f}} d t
$$


where $Q$ is the time-varying flowrate, $\tau$ is the time constant and $K_{p}$ and $K_{i}$ are proportional and integral gains, set as 3, 70 and 0.1 respectively in order to replicate the dynamics illustrated in Figure 4(b). For the present study it has been assumed that despite the variation in cerebral blood flow throughout the cardiac cycle, the autoregulation mechanism does not attempt to regulate to these higher frequency variations in blood flow. To simulate this feature the two terms on the right hand side of (8) comprise a proportionalintegral (PI) controller which has the effect of filtering out the higher frequency flowrate errors and causing the permeability to alter only in response to changes in the mean flowrate For incorporation with the continuity and momentum equations (8) has been discretized with a first order backwards difference.

\subsection{Model Boundary Conditions}

The boundary conditions chosen for the present study were the specification of systemic and venous pressures at the inlets and outlets of the afferent and efferent arteries respectively. The reason for this choice, as opposed to specifying velocity inlets and outflows, is that the specification of a velocity profile over the inlet fixes the flowrate through that inlet and does not allow the autoregulation mechanism to control blood flow correctly. For the efferent pressure outlets a venous pressure of $4 \mathrm{mmHg}$ was specified. For the present study, the pulsatile nature of the afferent blood pressure has been ignored as it is only the changes in mean cerebral blood flow that are of interest, hence the afferent pressure inlets are set at $100 \mathrm{mmHg}$.

The reference flowrates for the efferent cerebral arteries have been calculated by assuming a total flow into the circle of Willis of $12.5 \mathrm{~cm}^{3} \mathrm{~s}^{-1}$ with a peripheral resistance ratio of $6: 3: 4$ between the anterior, middle and posterior cerebral territories respectively (Hillen et al. 1986). Assuming that the blood flow through the anterior, middle and posterior is divided symmetrically between the left and right hemispheres, the reference flowrates can be calculated to be 1.39, 2.78 and $2.08 \mathrm{~cm}^{3} \mathrm{~s}^{-1}$ for the ACA, MCA and PCA respectively.

\section{Results}

For the three simulations performed an initial period of time was given under conditions of normal afferent blood pressure, with the models at the reference efferent flowrates, the purpose being to determine the relative importance of each arterial segment under conditions of normal afferent blood pressure. Subsequently an afferent pressure drop of $20 \mathrm{mmHg}$ was imposed in a single ICA (producing a reduction in afferent blood flow), to observe the collateral flow through the circle of Willis in order to return all efferent blood flowrates to their reference value and the differences between pathological conditions. The simulations were performed using the finite volume CFD package Fluent, with the autoregulation and Carreau-Yasuda code incorporated as a compiled user defined function. Each simulation ran for approximately 48 hours on a pentium 4 windows platform. The simulation results showed that generally, within a given pathological condition similar transient flowrate patterns occur. For brevity therefore the full transient flowrate plots for the efferent, afferent and communicating arteries are shown only for the the complete circle of Willis. The definition of the sign of the flowrate in the communicating arteries has been defined such that a positive flowrate in the $\mathrm{ACoA}$ is from the left to right side of the $\mathrm{CoW}$ and a positive flowrate in the PCoA's is from anterior to posterior.

\subsection{Normal Afferent Blood Pressure}

For the complete CoW under conditions of normal afferent blood pressure and with all the efferent arteries at their reference flowrates, the afferent blood supply is essentially split symmetrically between the left and right afferent arteries with the ICA's supplying almost three times as much blood to the cerebral territories as the VA's (Figure 5(a)). There is essentially no flow through the ACoA and only a relatively small equal amount of blood flow through the PCoA's (Figure 5(b)). The direction of blood flow through the PCoA's is from anterior to posterior indicating that the MCA's contribute to supplying blood to the posterior cerebral territories. For the fetal P1 condition more blood is supplied by the ICA's and less through the VA's compared to a complete CoW. In addition the supply is not split equally between the ICA's with the RICA (ipsilateral to the fetal P1) providing a greater afferent supply. There is also a small but significant amount of blood flow through the ACoA and almost three times as much blood flow through the RPCoA compared with a normal complete CoW. The reason for the increased afferent supply from the RICA and the RPCoA is that the increased resistance to blood flow through the RPCA P1 (the fetal P1) means that less afferent blood can be supplied to the RPCA through the VA's, hence requiring another route, namely 


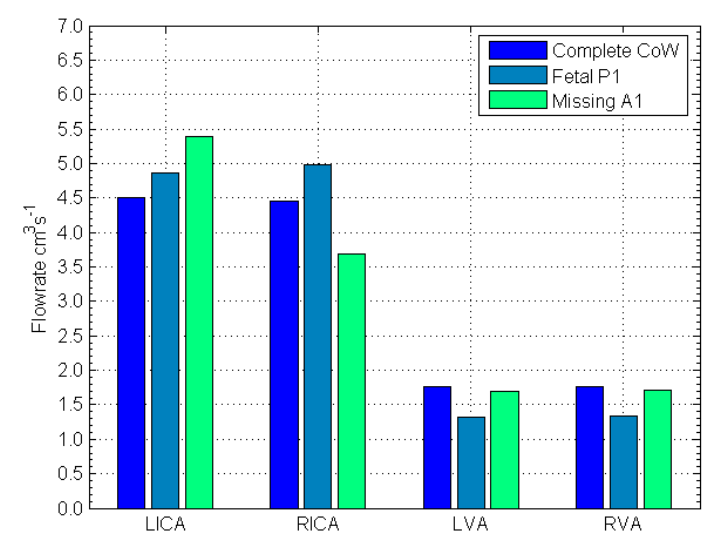

(a)

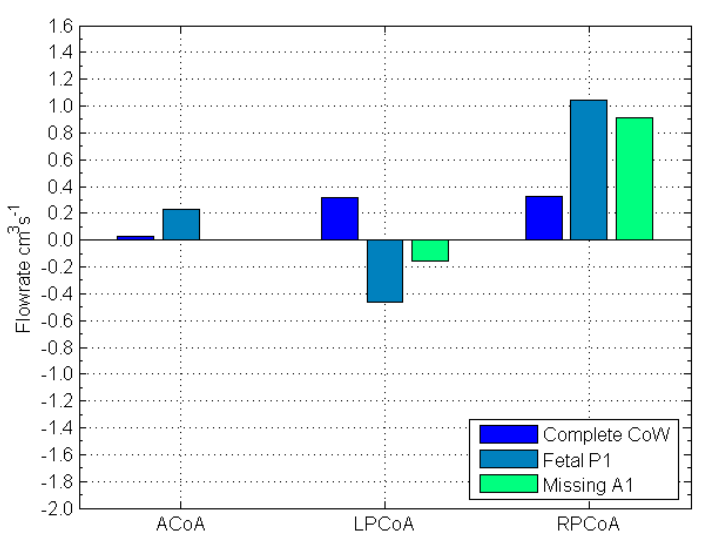

(b)

Figure 5: Initial (a) Afferent and (b) Communicating Artery Flowrates for the 3 CoW Pathological Conditions

the RICA and the RPCoA. Another notable feature is that blood flow through the LPCoA, while of a similar magnitude compared to a complete $\mathrm{CoW}$, flows from posterior to anterior indicating that the VA's contribute to supplying the left anterior cerebral territories. For the missing A1 the greatest asymmetry in afferent blood supply between the left and right sides of the CoW occurs. Due to the absence of the RACA A1, blood supply to the RACA must pass solely through the LACA A1 and there is hence significantly more blood supplied by the LICA than by the RICA. Blood supply through the VA's however is of a similar magnitude as for the complete CoW. Blood flow through the RPCoA is of a similar magnitude compared to that of the fetal P1 and flows from anterior to posterior. Blood flow through the LPCoA however, is the lowest in magnitude for all pathological conditions and flows from posterior to anterior. There is therefore blood supply re-routed around the posterior arteries to contribute blood to the left anterior of the CoW.

\subsection{Response to Afferent Pressure Drop}

Following the $20 \mathrm{mmHg}$ pressure drop in the RICA of the complete CoW there is an immediate reduction in blood flow through it (Figure 6(a)) and through all of the efferent arteries (Figure 6(c)) with the three efferent arteries ipsilateral to the pressure drop showing the greatest relative reduction. Intuitively the ipsilateral side experiences a reduction in blood flow because the supply from the RICA is reduced. The contralateral side experiences a reduction however because the alteration in blood pressure throughout the $\mathrm{CoW}$ causes an immediate increase in blood flow through the ACoA from left to right (drawing blood away from the LMCA and LACA) and through the RPCoA which reverses direction and flows from posterior to anterior (drawing blood away from the PCA's). In contrast blood flow through the LPCoA (contralateral to the pressure drop) is virtually unaffected. The pressure drop also causes an immediate initial increase in blood supply through the LICA and also through both of the VA's. For the remainder of the simulation the blood flow through the communicating arteries remains unchanged and the restoration of efferent blood flow is achieved by an increase in afferent blood supply from both ICA's and only a minor increase through the VA's. The reference flowrates adopted by the efferent arteries are lowered due to the reduction in arterial blood pressure. A notable feature illustrated in the streamline plot of Figure 6(d) is that blood flow through the A1 segment of the RACA is reduced essentially to zero. The RICA therefore contributes no blood supply to the RACA A2, instead this artery is supplied with blood solely through the ACoA.

The major difference in collateral flow for the fetal P1 condition is that due to the reduced diameter of the RPCA P1 segment, less blood is able to be re-routed through it. The result is that while blood flow through the RPCoA reverses direction to supply the RMCA, the actual amount of blood flow is substantially reduced compared to the complete $\mathrm{CoW}$ (Figure 7(b)). Furthermore, as the amount of blood flow through the RPCA P1 is reduced, the amount of blood flow through the VA's, while still increased from the flowrate under symmetric afferent blood pressure conditions, is much lower compared to a complete CoW 7(a)). The major difference in collateral flow for the missing A1 condition there is a large flow through the RPCoA 


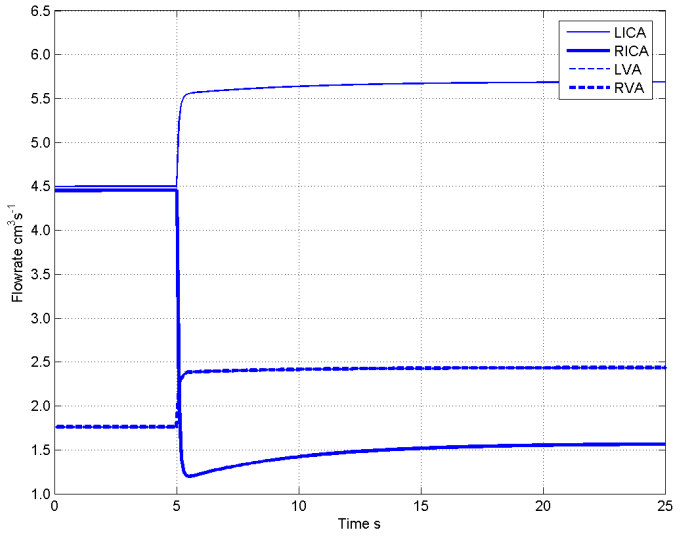

(a)

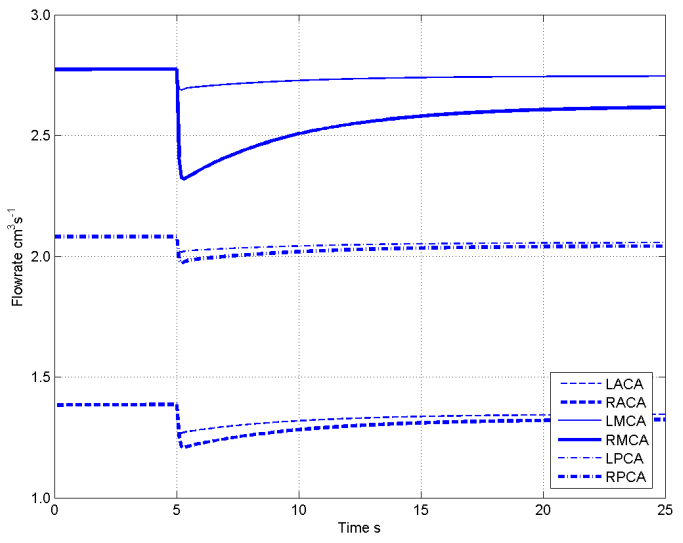

(c)

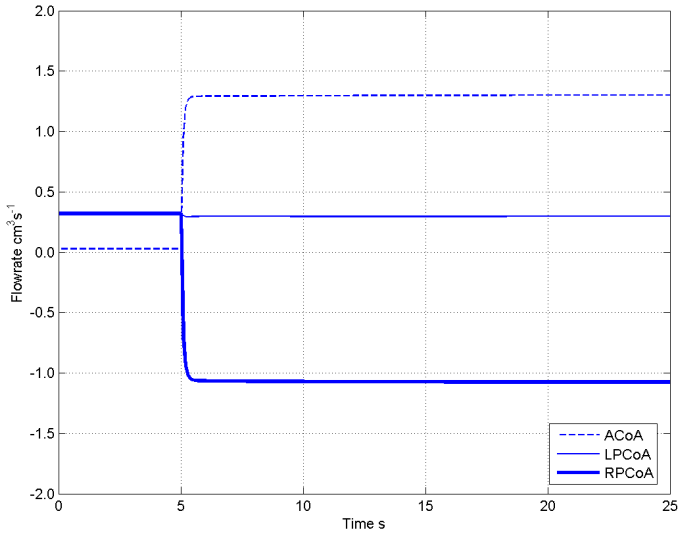

(b)

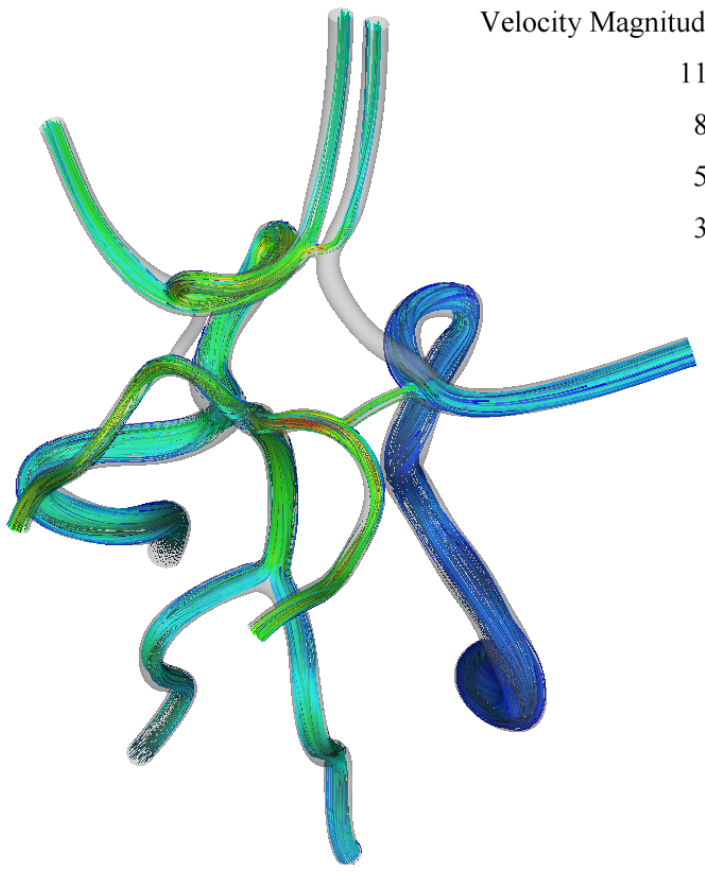

(d)

Figure 6: Complete CoW Transient Flowrates in the (a) Afferent, (b) Communicating and (c) Efferent Arteries and (d) Streamlines Following the Pressure Drop

from anterior to posterior, and also a large flow through the LPCoA from posterior to anterior, meaning that essentially, blood is being re-routed around the posterior of the CoW to contribute blood supply to the LMCA and the two ACA's.

\section{Discussion}

In developing a model of this complexity, validation is of the utmost importance if the model results are to be trusted. As it is blood flowrates through various cerebral arteries which are the major result, then the best form of validation is comparing the calculated model flowrates to in vivo experimental data. One technique commonly used to obtain quantitative information on cerebral blood flow is phase-contrast MRI. The basic principal behind this technique is that spins moving in the direction of a magnetic field gradient experience a phase shift which is proportional to the velocity. By summing the product of the pixel intensity 


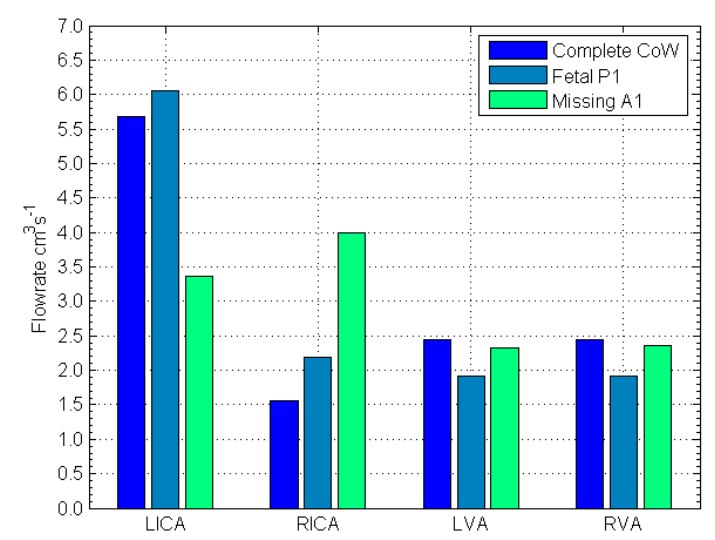

(a)

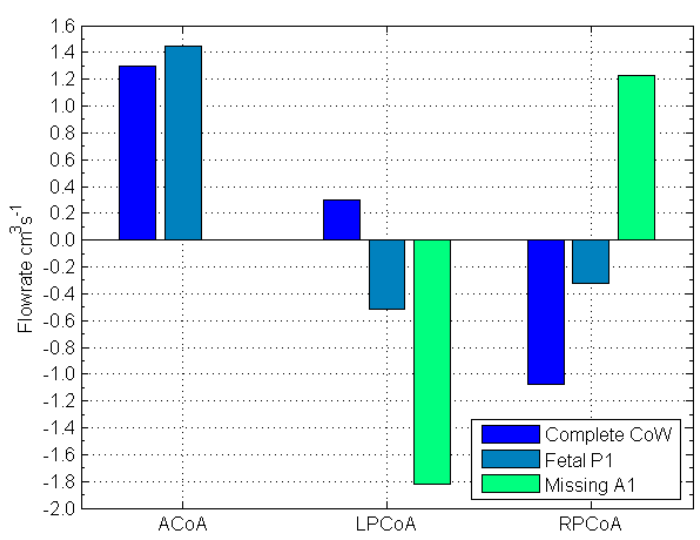

(b)

Figure 7: Final (a) Afferent and (b) Communicating Artery Flowrates for the 3 CoW Pathological Conditions

(representing velocity) and area for all of the pixels comprising the cross section of an artery, the flowrate can be calculated. A number of studies have been performed (Table 2) using this technique to generate data sets for human cerebral blood flow. While most of these studies only measure blood flow in the ICA's and the Basilar Artery (BA), some studies have been performed which measure blood flow in the six major cerebral arteries. To the authors knowledge however, no studies have been performed in which blood flow through the communicating arteries has been measured quantitatively. The studies performed generally include a wide range of age groups to which cerebral blood flow is strongly related and furthermore the studies make no distinction between differing pathological conditions of the circle of Willis, making the validation of the differences in blood flow between pathological conditions difficult and However, the data sets are still a useful validation to show that the model results are within acceptable physiological ranges.

Table 2: Cerebral Blood Flow Studies Using Phase-Contrast MRI

\begin{tabular}{|c|c|c|c|c|c|c|c|c|c|}
\hline & \multicolumn{9}{|c|}{ Volume Flowrate $\mathrm{cm}^{3} \mathrm{~s}^{-1}$ mean $\pm \mathrm{SD}$} \\
\hline Study & LACA & RACA & LMCA & RMCA & LPCA & RPCA & LICA & RICA & $\overline{B A}$ \\
\hline $\begin{array}{l}\text { Current Model } \\
\text { (Complete CoW) }\end{array}$ & 1.39 & 1.39 & 2.78 & 2.78 & 2.08 & 2.08 & 4.50 & 4.50 & 3.50 \\
\hline (Enzmann et al. 1994) & $\begin{array}{r}1.25 \\
\pm 0.17\end{array}$ & $\begin{array}{r}1.47 \\
\pm 0.18\end{array}$ & $\begin{array}{r}1.80 \\
\pm 0.12\end{array}$ & $\begin{array}{r}2.12 \\
\pm 0.12\end{array}$ & $\begin{array}{r}0.88 \\
\pm 0.07\end{array}$ & $\begin{array}{r}0.85 \\
\pm 0.07\end{array}$ & $\begin{array}{r}5.62 \\
\pm 0.32\end{array}$ & $\begin{array}{r}5.03 \\
\pm 0.35\end{array}$ & $\begin{array}{r}2.68 \\
\pm 0.18\end{array}$ \\
\hline (Marks et al. 1992) & & & & & & & $\begin{array}{r}5.70 \\
\pm 0.25\end{array}$ & $\begin{array}{r}5.87 \\
\pm 0.35\end{array}$ & $\begin{array}{r}2.73 \\
\pm 0.20\end{array}$ \\
\hline (Buijs et al. 1998) & & & & & & & $\begin{array}{r}4.12 \\
\pm 1.20\end{array}$ & $\begin{array}{r}4.17 \\
\pm 1.15\end{array}$ & $\begin{array}{r}2.00 \\
\pm 0.78\end{array}$ \\
\hline (Obata et al. 1996) & & & & & & & $\begin{array}{r}3.82 \\
\pm 1.43\end{array}$ & $\begin{array}{r}3.72 \\
\pm 0.97\end{array}$ & $\begin{array}{r}2.37 \\
\pm 0.97\end{array}$ \\
\hline
\end{tabular}

The afferent flowrates through the ICA's and the efferent flowrates through the ACA's agree reasonably well with experimental data. The flowrate through the BA, MCA's and most noticeably the PCA's however, appears to have been overestimated slightly for the present study. A likely explanation for the overestimation of blood flow through the various arterial segments is due to the absence of a number of arteries that branch off the afferent ICA's and BA's. In particular the Ophthalmic and Anterior Choroidal arteries that branch off each ICA and would reduce the amount of blood flow passing through the MCA's. Also the Anterior Inferior Cerebellar and Superior Cerebellar arteries that branch off the BA would reduce the blood flow passing through the BA and PCA's. The apparent lack of symmetry between the left and right sides of the 
CoW is likely caused by the inclusion of different pathological conditions within the datasets.

The simulations performed in this study explored three common pathological conditions of the circle of Willis. There are many other conditions which exist (Alpers et al. 1959) and in addition, variations in the relative diameters of the arteries within given condition (Table 1). Combined with varying levels of stenosis in one or multiple afferent arteries there is an extremely large number of possible simulations which could be performed to investigate effect of the variability of circle of Willis geometry on the cerebral hemodynamics. This study makes no attempt to cover these combinations but rather to present the model development and some basic results. As the end goal of the research is for a clinical tool, it is likely that in future work the model will be applied to patient specific circle of Willis models, segmented directly from MRI data of an individuals cerebral vasculature. In addition, phase-contrast MRI scans performed on an individual at the same time as the TOF scan (used to generate the geometry) may be used to validate the model results more accurately.

\section{References}

Alpers, B. J., R. G. Berry, and R. M. Paddison (1959). Anatomical studies of the circle of willis in normal brain. AMA Arch Neurol Psychiatry 81(4), 409-18.

Buijs, P. C., M. J. Krabbe-Hartkamp, C. J. G. Bakker, E. E. de Lange, L. M. P. Ramos, M. M. B. Breteler, and W. P. T. M. Mali (1998). Effect of age on cerebral blood flow: Measurement with ungated twodimensional phase-contrast $\mathrm{mr}$ angiography in 250 adults. Radiology $209(3), 667-674$.

Cassot, F., M. Zagzoule, and J. P. Marc-Vergnes (2000). Hemodynamic role of the circle of willis in stenoses of internal carotid arteries. an analytical solution of a linear model. J Biomech 33(4), 395-405.

Cebral, J. R., M. A. Castro, O. Soto, R. Lohner, and N. Alperin (2003). Blood-flow models of the circle of willis from magnetic resonance data. Journal of Engineering Mathematics 47(3-4), 369-386.

Chen, J. and X. Y. Lu (2004). Numerical investigation of the non-newtonian blood flow in a bifurcation model with a non-planar branch. J Biomech 37(12), 1899-911.

David, T., M. Brown, and A. Ferrandez (2003). Auto-regulation and blood flow in the cerebral circulation. International Journal for Numerical Methods in Fluids 43(6-7), 701-713.

Enzmann, D. R., M. R. Ross, M. P. Marks, and N. J. Pelc (1994). Blood-flow in major cerebral-arteries measured by phase-contrast cine mr. American Journal of Neuroradiology 15(1), 123-129.

Ferrandez, A., T. David, and M. D. Brown (2002). Numerical models of auto-regulation and blood flow in the cerebral circulation. Comput Methods Biomech Biomed Engin 5(1), 7-19.

Gijsen, F. J., F. N. van de Vosse, and J. D. Janssen (1999). The influence of the non-newtonian properties of blood on the flow in large arteries: steady flow in a carotid bifurcation model. $J$ Biomech 32(6), 601-8.

Guyton, A. C. and J. E. Hall (2000). Textbook of medical physiology (10th ed.). Philadelphia: Saunders.

Hillen, B., H. W. Hoogstraten, and L. Post (1986). A mathematical model of the flow in the circle of willis. J Biomech 19(3), 187-94.

Keener, J. P. and J. Sneyd (1998). Mathematical physiology. Interdisciplinary applied mathematics ; v. 8. New York: Springer.

Marks, M. P., N. J. Pelc, M. R. Ross, and D. R. Enzmann (1992). Determination of cerebral blood-flow with a phase-contrast cine $\mathrm{mr}$ imaging technique - evaluation of normal subjects and patients with arteriovenous-malformations. Radiology 182(2), 467-476.

Moorhead, K. T., C. V. Doran, J. G. Chase, and T. David (2004). Lumped parameter and feedback control models of the auto-regulatory response in the circle of willis. Comput Methods Biomech Biomed Engin 7(3), 121-30.

Newell, D. W., R. Aaslid, A. Lam, T. S. Mayberg, and H. R. Winn (1994). Comparison of flow and velocity during dynamic autoregulation testing in humans. Stroke 25(4), 793-797.

Obata, T., F. Shishido, M. Koga, H. Ikehira, F. Kimura, and K. Yoshida (1996). Three-vessel study of cerebral blood flow using phase-contrast magnetic resonance imaging: Effect of physical characteristics. Magnetic Resonance Imaging 14(10), 1143-1148. 
Olufsen, M. S., A. Nadim, and L. A. Lipsitz (2002). Dynamics of cerebral blood flow regulation explained using a lumped parameter model. Am J Physiol Regul Integr Comp Physiol 282(2), R611-22.

Petty, G. W., R. D. Brown, J. P. Whisnant, J. D. Sicks, W. M. O'Fallon, and D. O. Wiebers (2000). Ischemic stroke subtypes - a population-based study of functional outcome, survival, and recurrence. Stroke 31(5), 1062-1068.

Ursino, M. (1991). A mathematical-model of overall cerebral blood-flow regulation in the rat. Ieee Transactions on Biomedical Engineering 38(8), 795-807.

Ursino, M. and M. Giulioni (2003). Quantitative assessment of cerebral autoregulation from transcranial doppler pulsatility: a computer simulation study. Med Eng Phys 25(8), 655-66. 